\title{
Semidisquotation and the infinitary function of truth
}

\author{
Camillo Fiore
}

February 22, 2021

\begin{abstract}
The infinitary function of the truth predicate consists in its ability to express infinite conjunctions and disjunctions. A transparency principle for truth states the equivalence between a sentence and its truth predication; it requires an introduction principle - which allows the inference from "snow is white" to "the sentence "snow is white' is true" - and an elimination principle - which allows the inference from "the sentence 'snow is white' is true" to "snow is white". It is commonly assumed that a theory of truth needs to satisfy a transparency principle to fulfil the infinitary function. Picollo and Schindler (Erkenntnis 83:899-928, 2017) argue against this idea. They prove that, given certain assumptions, an elimination principle is sufficient for the purpose. Then, they pose a challenge: to show why we should incorporate introduction principles to our theory of truth. In this essay I take on the challenge. I show that, given the authors' assumptions, an introduction principle is also sufficient to perform the infinitary function.
\end{abstract}

\section{Introduction}

For many authors, the truth predicate plays an expressive role of a quasi-logical nature: ${ }^{1}$ it allows us to express, in a first-order language, conjunctions and disjunctions of infinitely many sentences. If $P$ is a predicate of sentences and there are infinitely many $P$ s, it is said that "All $P$ s are true" expresses the infinite conjunction of all $P$ s while "Some $P$ s are true" expresses their infinite disjunction (cf. Gupta 1993; Leeds 1978; Putnam 1978; Quine 1970). I will refer to this as the infinitary function of truth.

For many, the truth predicate also plays an expressive role with epistemic and rhetoric uses: it allows us to express one or more sentences avoiding their explicit articulation. If we want to assert, e.g. Maxwell's equations, but we do not know them-or we want to save space or time, or an explicit assertion is conversationally inappropriate, and so on and so forth - we can express all four of them by just saying "Maxwell's equations are true". This is known as the blind ascription function of truth.

Of the roles mentioned, the logical interest of truth lies, arguably, in the infinitary function alone. In first-order languages, finite conjunctions and disjunctions are always in principle expressible without the truth predicate; in contrast, many infinite conjunctions and disjunctions

\footnotetext{
${ }^{1}$ It is not logical simpliciter because a theory of truth requires a background theory about the objects to which truth is applied (e.g. sentences, Gödel codes thereof, propositions), that is, a theory of syntax.
} 
cannot be expressed without it. The infinitary function increases the expressive power of our first-order languages, and thus encourages the formulation of so-called 'logics' of truth-more precisely, formal theories thereof.

Let $\mathcal{L}$ be a first-order language with identity and $\mathcal{L}_{T}$ the result of expanding $\mathcal{L}$ with a monadic predicate symbol $T$ for truth. $\mathcal{L}$ contains a term $\ulcorner\sigma\urcorner$ denoting $\sigma$ (perhaps via some coding) for each expression $\sigma$ of $\mathcal{L}_{T}$. Let $\Sigma \subseteq \mathcal{L}$ be our base theory. We assume $\Sigma$ is able to prove elementary facts about the syntax of $\mathcal{L}_{T} .{ }^{2}$ A theory of truth will be any (recursively enumerable) subset of $\mathcal{L}_{T}$ that contains the base theory $\Sigma$ and is closed under first-order (not necessarily classical) logical consequence.

Our driving question is: what principles (axioms or rules of inference) do we need to postulate for $T$ to fulfil the infinitary function? The most common answer is that we need a principle that states the equivalence between a sentence $\phi$ and its truth predication $T\ulcorner\phi\urcorner$. In the truth-theoretic literature, this is known as a transparency or disquotation principle. It requires an introduction half — which allows the inference from $\phi$ to $T\ulcorner\phi\urcorner$ —and an elimination half-which allows the inference from $T\ulcorner\phi\urcorner$ to $\phi$. It can be given by all instances of the well known equivalence

$$
T\ulcorner\phi\urcorner \leftrightarrow \phi
$$

where $\phi$ is a sentence of $\mathcal{L}$. But one can also use a pair of rules, such as

$$
\begin{aligned}
& \phi \vdash T\ulcorner\phi\urcorner \\
& T\ulcorner\phi\urcorner \vdash \phi
\end{aligned}
$$

where the inferences from $\phi$ to $T\ulcorner\phi\urcorner$ and vice versa are allowed even in hypothetical contexts. (These rules should not be confused with NEC and CONEC, which allow the mentioned inferences only given a proof of the premise (cf. Halbach 2011, p. 152). $)^{3}$

Unfortunately, Tarski (1935) proved that an unrestricted transparency principle trivialises any classical theory that proves our syntactical background assumptions. This result has led to two different kinds of proposals: either we (i) reject some instances of our transparency principle (e.g. Horwich 1998), or we (ii) reject classical logic, and adopt some alternative system that can be structural (e.g. Beall 2009; Field 2008; Kripke 1975; Priest 2006) or substructural (e.g. Cobreros et al. 2013; Da Ré 2020; Rosenblatt 2019; Zardini 2011).

Picollo and Schindler (2017) argue against the common idea that the infinitary function requires a transparency principle. They entertain certain assumptions concerning what it means to express infinite conjunctions and disjunctions. They prove that an elimination principle (such as T-Elim) is sufficient for the purpose; viz. an introduction principle (such as T-Intro) is not needed. An unrestricted elimination principle can consistently extend classical logic. Hence, it seems that we could dispense with introduction principles and obtain a type-free, ${ }^{4}$ classical theory of truth that fulfils the infinitary function. The authors conclude that an elimination principle "should be part of any reasonable theory of truth" (p. 919) and they pose

\footnotetext{
${ }^{2}$ In the literature, Peano or Robinson arithmetic are usually taken as syntax theory. At any case, we assume that $\Sigma$ interprets some decent amount of arithmetic.

${ }^{3}$ The schema and the pair of rules are equivalent in classical logic. However, the equivalence does not hold in every non-classical system, so there could be reasons to prefer one transparency principle over the other.

${ }^{4} \mathrm{~A}$ truth theory is said to be type-free if it proves sentences of the form $T\ulcorner T\ulcorner\phi\urcorner\urcorner$.
} 
a challenge "for all those that believe that introduction principles for truth must be part of a logic of truth" (p. 914).

In this essay I take on the challenge. I do not argue that the infinitary function requires a transparency principle. However, I claim that, given the authors' assumptions, elimination principles do not play a privileged role in the infinitary function, and it is not the case that every 'reasonable' theory of truth should incorporate them.

I call the proposal offered by Picollo and Schindler semidisquotational, because it consists in a type-free theory of truth that validates at least an elimination principle. It can be given either by the rule T-Elim, or by all instances of

$$
T\ulcorner\psi\urcorner \rightarrow \psi
$$

I define an alternative semidisquotational proposal that validates at least an introduction principle, given either by the rule T-Intro, or by all instances of

$$
\psi \rightarrow T\ulcorner\psi\urcorner
$$

I show that, given the authors' assumptions, the introduction-based proposal also suffices to fulfil the infinitary function. Moreover, I argue that it does the job as well as its eliminationbased counterpart. My conclusion is a conditional statement: if we want to reject transparency, then, as regards the infinitary function, there seem to be no reasons of logical nature to choose between introduction and elimination principles.

A word of caution. I wish not to make either a defence or an absolute assessment of any of the semidisquotational proposals considered. I do not advocate the thesis that an introduction or elimination principle is sufficient to fulfil the infinitary function. My more modest goal is to compare the relative merits of the proposals considered and conclude, against Picollo and Schindler, that the one based on introduction principles is just as good.

The plan is simple. In Sect. 2, I introduce Picollo and Schindler's proposal. In Sect. 3, I define and defend the introduction-based alternative. In the Appendix, I proof some of the technical claims I make in the essay.

\section{Elimination's supremacy}

I start with a brief summary of Picollo and Schindler's semidisquotational proposal.

Consider any infinite lot of sentences. If it can be obtained by uniformly substituting closed terms for variables in some standard formula - e.g. " $x$ is divisible by 2 " - and the class of the objects all those terms denote is definable in the language by a suitable predicate - e.g. " $x$ is an even number" - then the infinite conjunction (disjunction) of all the sentences can be expressed by mere universal (existential) quantification - e.g. "All even numbers are divisible by 2". When the infinite lot of sentences cannot be obtained by substitution as described, however, the truth predicate comes into play. (Cf. Quine 1970, chap. 1.) 


\section{$2.1 \quad$ Infinite conjunctions}

Let $\phi(x)$ be a predicate of $\mathcal{L}_{T}$-sentences and suppose there are infinitely many $\phi \mathrm{s}^{5}{ }^{5}$ The conjunction of all the $\phi$ s is the conjunction of all the instances of the schema $\phi(\ulcorner\psi\urcorner) \rightarrow \psi$, namely, $\bigwedge\left\{\phi(\ulcorner\psi\urcorner) \rightarrow \psi: \psi \in \mathcal{L}_{T}\right\}$.

The usual sentence to express an infinite conjunction is

$$
\forall x(\phi(x) \rightarrow T x)
$$

That is, the orthodox formalisation of "All $\phi$ s are true".

Picollo and Schindler focus on a broadly conceived finite-axiomatisation approach. Inf-C is said to express an infinite conjunction $\bigwedge\left\{\phi(\ulcorner\psi\urcorner) \rightarrow \psi: \psi \in \mathcal{L}_{T}\right\}$ in a theory of truth $\Gamma$ if and only if everything deducible in $\Gamma$ from the set $\left\{\phi(\ulcorner\psi\urcorner) \rightarrow \psi: \psi \in \mathcal{L}_{T}\right\}$ is also deducible in $\Gamma$ from Inf-C. ${ }^{6}$

The authors point out that, given some assumptions on the classicality of our background logic $\vdash$, an elimination principle is sufficient to validate the inference

$$
\forall x(\phi(x) \rightarrow T x), \phi(\ulcorner\psi\urcorner) \vdash \psi
$$

Observation 1 (Picollo and Schindler). Let $\Gamma \subseteq \mathcal{L}_{T}$ be a truth theory where T-Elim or T-Out holds, formulated over a logic in which the rules of Universal Instantiation and Modus Ponens are valid. Then, (1) holds in $\Gamma$.

If the deduction theorem holds for $\vdash,(1)$ ensures that

$$
\forall x(\phi(x) \rightarrow T x) \vdash \phi(\ulcorner\psi\urcorner) \rightarrow \psi
$$

If $\vdash$ is transitive, it follows that everything deducible from the infinitely many instances of $\phi(\ulcorner\psi\urcorner) \rightarrow \psi$ is also deducible from $\forall x(\phi(x) \rightarrow T x)$, that is to say, that Inf-C expresses an infinite conjunction.

The authors consider an objection. Predicates satisfying T-Out and T-Elim, such as $x \neq x$, are definable in any classical theory. But if we interpret $T x$ as $x \neq x$, then Inf-C is inconsistent as long as $\exists x \phi(x)$ is provable, and this is not how conjunctions should behave. Hence, T-Out or T-Elim cannot be enough for Inf-C to express an infinite conjunction.

The authors' reply — as I understand it - is that T-Out and T-Elim are not enough indeed. We also assume that $T$ stands for a truth predicate. Axioms governing a truth predicate should be consistent with the assumption that there is at least one true sentence. Hence, $x \neq x$ does not stand for a truth predicate, and if we interpret $T x$ as $x \neq x$, we are not compelled to admit that Inf-C expresses an infinite conjunction. In the authors' words, "a truth predicate governed by T-Out is not definable in or reducible to the base theory" (p. 912).

\footnotetext{
${ }^{5} \mathrm{By}$ "Let $\phi(x)$ be a predicate of $\mathcal{L}_{T}$-sentences", I mean that the syntax theory $\Sigma$ proves the (formalisation of) the claim "For all $x$, if $\phi(x)$ then $x$ is a sentence of $\mathcal{L}_{T}$ ". (I follow Picollo and Schindler on this assumption (p. 907).)

${ }^{6}$ The qualification 'broadly conceived' is meant to distinguish this proposal from the one in Halbach (1999). (See Sect. 3.4.)
} 


\subsection{Infinite disjunctions ${ }^{7}$}

Let $\phi(x)$ be a predicate of $\mathcal{L}_{T}$-sentences and suppose there are infinitely many $\phi \mathrm{s}$. The disjunction of all the $\phi \mathrm{s}$ is the disjunction of all the instances of the schema $\phi(\ulcorner\psi\urcorner) \wedge \psi$, namely, $\bigvee\left\{\phi(\ulcorner\psi\urcorner) \wedge \psi: \psi \in \mathcal{L}_{T}\right\}$.

The usual sentence to express an infinite disjunction is

$$
\exists x(\phi(x) \wedge T x)
$$

That is, the orthodox formalisation of "Some $\phi$ s are true".

Picollo and Schindler adopt two assumptions advanced by Halbach (1999). The assumptions amount to the following: (a) the negation of the infinite disjunction of the $\phi$ s, viz. $\neg \bigvee\{\phi(\ulcorner\psi\urcorner) \wedge$ $\left.\psi: \psi \in \mathcal{L}_{T}\right\}$, is equivalent to the infinite conjunction of the negation of the $\phi$ s, viz. $\bigwedge\{\phi(\ulcorner\psi\urcorner) \rightarrow$ $\left.\neg \psi: \psi \in \mathcal{L}_{T}\right\}$, in the sense that they have the same $\mathcal{L}_{T}$-consequences over a truth theory $\Gamma$, and (b) an infinite disjunction is expressed by a truth-theoretic sentence $\xi$ if and only if the negation of the disjunction is expressed by $\neg \xi$.

Given these assumptions and the finite-axiomatisation approach defined in Sect. 2.1, an elimination principle is not sufficient for Inf-D to express an infinite disjunction. Nevertheless, the authors offer an alternative sentence: ${ }^{8}$

$$
\neg \forall x(\phi(x) \rightarrow T \neg x)
$$

By a small modification of our previous argument, an elimination principle is sufficient for the negation of Inf-D* to express the conjunction $\bigwedge\left\{\phi(\ulcorner\psi\urcorner) \rightarrow \neg \psi: \psi \in L_{T}\right\}$. By assumption (a), such conjunction is equivalent to $\neg \bigvee\left\{\phi(\ulcorner\psi\urcorner) \wedge \psi: \psi \in L_{T}\right\}$. Then, by assumption (b), Inf-D* expresses an infinite disjunction.

Moreover, suppose that identity behaves classically, ${ }^{9}$ and $\vdash$ validates the usual rules of conjunction introduction, negation introduction, Universal Instantiation and Modus Ponens. Then, an elimination principle is sufficient to validate the inference

$$
\phi(\ulcorner\psi\urcorner), \psi \vdash \neg \forall x(\phi(x) \rightarrow T\urcorner x)
$$

which is regarded as an intuitive requirement for the sentence on the right-hand side of the turnstile to express an infinite disjunction.

In conclusion: given the approach in Sect. 2.1 and assumptions (a) and (b), infinite conjunctions and disjunctions are expressible by means of elimination principles alone. Besides, elimination principles can consistently extend classical logic. Hence, it seems that we can dispense with introduction principles - thereby abandoning transparency - and obtain a type-free, classical theory that fulfils the infinitary function. ${ }^{10}$

\footnotetext{
${ }^{7}$ In their article, Picollo and Schindler show that an elimination principle is sufficient to express infinite disjunctions relative to the finite-axiomatisation approach in Halbach (1999). For the sake of uniformity, I make the argument from this section relative to the approach defined in Sect. 2.1. Accordingly, I adapt assumptions (a) and (b) by Halbach: in the original paper, they are restricted to infinite conjunctions and disjunctions of $T$-free sentences.

${ }^{8}$ We use $\neg$ to denote the negation function that, applied to (the code of) a formula $\phi$, gives (the code of) $\neg \phi$. We assume that the base theory $\Sigma$ proves $\neg\ulcorner\phi\urcorner=\ulcorner\neg \phi\urcorner$ for any formula $\phi$ of $\mathcal{L}_{T}$.

${ }^{9}$ This is, the inference from $s=t$ and $\phi(s)$ to $\phi(t)$ holds for every formula $\phi(x)$ and terms $s, t$.

${ }^{10}$ It is noteworthy that Picollo and Schindler do not commit themselves to the idea that the approach defined
} 


\section{Introduction's vindication}

Picollo and Schindler eventually conclude that an elimination principle "should be part of any reasonable theory of truth" (p. 919), whereas "the arguments for adopting introduction principles are not entirely convincing" (914). Then, they pose the mentioned challenge: to show why we should incorporate introduction principles to our theory of truth. In this section I take on the challenge. In Sect. 3.1, I define an alternative semidisquotational approach, relying only on introduction principles. In Sects. 3.2 to 3.4, I defend the approach and argue that it is as good as the elimination-based rival.

\subsection{Introduction is also enough}

We will mimic the strategy adopted by Picollo and Schindler.

Infinite conjunctions. An introduction principle is not sufficient to validate inference (1) in classical logic. Thus, we cannot run the argument from Sect. 2.1 as it stands. Just as Picollo and Schindler had to reject Inf-D, we have to reject Inf-C. Nevertheless, we offer an alternative sentence:

$$
\neg \exists x(\phi(x) \wedge T \neg x)
$$

By analogy with the criterion adopted by the authors, we say that Inf- $\mathrm{C}^{*}$ expresses an infinite conjunction $\bigwedge\left\{\phi(\ulcorner\psi\urcorner) \rightarrow \psi: \psi \in \mathcal{L}_{T}\right\}$ in a truth theory $\Gamma$ if and only if everything deducible in $\Gamma$ from the set $\left\{\phi(\ulcorner\psi\urcorner) \rightarrow \psi: \psi \in \mathcal{L}_{T}\right\}$ is also deducible in $\Gamma$ from Inf-C*. ${ }^{11}$

We point out that, given some assumptions on the classicality of $\vdash$, an introduction principle is sufficient to validate

$$
\neg \exists x(\phi(x) \wedge T \neg x), \phi(\ulcorner\psi\urcorner) \vdash \psi
$$

Observation 2. Let $\Gamma \subseteq \mathcal{L}_{T}$ be a truth theory where T-In or T-Intro holds, formulated over a logic where identity behaves classically, and the usual rules of conjunction introduction, negation introduction, introduction of the existential quantifier, Modus Ponens and double negation elimination are valid. Then, (3) holds in $\Gamma$.

Proof. Assume $\neg \exists x(\phi(x) \wedge T \neg x)$ and $\phi(\ulcorner\psi\urcorner)$. For reductio, suppose $\neg \psi$. By T-Intro or, alternatively, T-In and Modus Ponens, we get $T\ulcorner\neg \psi\urcorner$. By the laws of identity and the fact $\neg\ulcorner\phi\urcorner=\ulcorner\neg \phi\urcorner$ provable in the base theory, we get $T \neg\ulcorner\psi\urcorner$. We introduce both the conjunction and the existential quantifier and obtain $\exists x(\phi(x) \wedge T \neg x)$. This contradicts one of our initial assumptions, so we conclude $\neg \neg \psi$. The elimination of double-negation delivers $\psi$.

in Sect. 2.1, together with assumptions (a) and (b), give a satisfactory account on the expressive functions of truth. They put forward a different account in (2019).

${ }^{11}$ I do not assume, and do not attribute to Picollo and Schindler, the view that any sentence expresses the conjunction of all the $\phi$ s just in case it proves all the consequences of the conditionals $\phi(\ulcorner\psi\urcorner) \rightarrow \psi$. (I thank an anonymous reviewer for suggesting me to clarify this.) Two points are worth mentioning. First, Inf-C and Inf$\mathrm{C}^{*}$ differ from a contradiction in that, if $T$ is a primitive predicate, they do not prove the conditionals mentioned by classical logic alone. Second, Inf-C and Inf-C* differ from most sentences in that, when combined with the appropriate half of a transparency principle, they have some interesting properties regarding the conservativity of the theory of truth (see Sect. 3.4). 
Again, if $\vdash$ is transitive and satisfies the deduction theorem, (3) ensures that everything deducible from the infinitely many instances of $\phi(\ulcorner\psi\urcorner) \rightarrow \psi$ is also deducible from $\neg \exists x(\phi(x) \wedge$ $T \neg x)$, that is to say, that Inf-C* expresses an infinite conjunction.

The worry mentioned in Sect. 2.1 arises again here. Predicate $x=x$ satisfies T-In and T-Intro in any classical theory. But if we interpret $T x$ as $x=x$, then Inf-C* is inconsistent as long as $\exists x \phi(x)$ is provable. Hence, T-In or T-Intro cannot be enough for Inf-C* to express an infinite conjunction.

My analogous reply is that T-In and T-Intro are not enough indeed. We also assume that $T$ stands for a truth predicate. Axioms for a truth predicate should be consistent with the assumption that at least one sentence is not true. Hence, $x=x$ does not stand for a truth predicate, and if we interpret $T x$ as $x=x$, we are not compelled to admit that Inf- $\mathrm{C}^{*}$ expresses an infinite conjunction.

Infinite disjunctions. By a small modification of our previous argument, an introduction principle is sufficient for the negation of Inf-D to express the conjunction $\bigwedge\{\phi(\ulcorner\psi\urcorner) \rightarrow \neg \psi$ : $\left.\psi \in \mathcal{L}_{T}\right\}$. By assumption (a), such conjunction is equivalent to $\neg \bigvee\left\{\phi(\ulcorner\psi\urcorner) \wedge \psi: \psi \in \mathcal{L}_{T}\right\}$. By assumption (b), Inf-D expresses an infinite disjunction.

Moreover, suppose that $\vdash$ validates the usual rules of conjunction introduction and introduction of the existential quantifier. Then, an introduction principle is sufficient to validate the inference

$$
\phi(\ulcorner\psi\urcorner), \psi \vdash \exists x(\phi(x) \wedge T x)
$$

thereby satisfying the intuitive requirement mentioned earlier (Sect. 2.2).

Introduction principles can also consistently extend classical logic. Hence, it seems that we can dispense with elimination principles and get a type-free, classical theory that fulfils the infinitary function all the same.

\subsection{A game of (counter)arguments}

Picollo and Schindler analyse two objections that seem to support the need for introduction principles for truth.

A) Consider the statement "If everything that Jack said is true, then $\psi$ ". Its formalisation is

$$
\forall x(\chi(x) \rightarrow T x) \rightarrow \psi
$$

where $\chi(x)$ applies exactly to the sentences uttered by Jack. Now suppose that what Jack said is exactly $\psi_{1}, \ldots, \psi_{n} .{ }^{12}$ We want this information, together with (5), to imply

$$
\left(\psi_{1} \wedge \ldots \wedge \psi_{n}\right) \rightarrow \psi
$$

But (6) will not be derivable unless we have an introduction principle.

B) Consider the thesis that every truth is knowable:

$$
\forall x(T x \rightarrow \diamond K x)
$$

\footnotetext{
${ }^{12}$ That is to say, suppose that $\forall x\left(\chi(x) \rightarrow\left(x=\left\ulcorner\psi_{1}\right\urcorner \vee \ldots \vee x=\left\ulcorner\psi_{n}\right\urcorner\right)\right)$
} 
where $K x$ means that $x$ is known and $\diamond$ is the possibility operator. We want (7) to imply every instance of

$$
\psi \rightarrow \diamond K\ulcorner\psi\urcorner
$$

But without an introduction principle, no instance of (8) will be derivable.

The authors reply with clever counterarguments. Let $\Gamma$ be a classical theory of truth with at least an elimination principle. In objection A, replace (5) by

$$
\forall x(\phi(x) \rightarrow T x)
$$

(that is, an instance of Inf-C) where $\phi(x)$ means ' $x$ is the unique sentence obtained by concatenating the conjunction of the $\chi_{\mathrm{s}}$ with ' $\rightarrow \psi$ ". Sentence (6) follows from $\left(5^{*}\right)$ plus the information relative to the content of $\chi$. In objection B, the strategy is similar: replace (7) by a sentence Inf-C where $\phi(x)$ means ' $x$ is an instance of (8)'. Each instance of (8) will follow. In general, we can express all the instances of a schema '.... ...', for any sentence $\psi$ of the language, using a sentence Inf-C where $\phi(x)$ means ' $x$ is an instance of schema '.......".

The authors conclude that, as regards the expressive power of truth, no convincing reasons to admit introduction principles have been given yet. However, one may put forward analogous objections to the introduction-based account:

c) Consider the statement "If $\psi$, then everything that Jack said is true". Its formalisation is

$$
\psi \rightarrow \forall x(\chi(x) \rightarrow T x)
$$

Suppose that what Jack said is exactly $\psi_{1}, \ldots, \psi_{n}$. We want this information, together with (9), to imply

$$
\psi \rightarrow\left(\psi_{1} \wedge \ldots \wedge \psi_{n}\right)
$$

But (10) will not be derivable unless we have an elimination principle.

D) Consider the thesis that knowledge implies truth:

$$
\forall x(K x \rightarrow T x)
$$

We want (11) to imply every instance of

$$
K\ulcorner\psi\urcorner \rightarrow \psi
$$

But without an elimination principle, no instance of (12) will be derivable.

And now the counterarguments. Let $\Gamma$ be a classical theory of truth with at least an introduction principle. In objection C, replace (9) by

$$
\neg \exists x(\phi(x) \wedge T \neg x)
$$

(that is, an instance of Inf- $\mathrm{C}^{*}$ ) where $\phi(x)$ means ' $x$ is the unique sentence obtained by concatenating ' $\psi \rightarrow$ ' with the conjunction of all the $\chi \mathrm{s}$ '. Sentence (10) follows from $\left(9^{*}\right)$ plus the information relative to the content of $\chi$. In objection D, replace (11) by a sentence Inf-C* where $\phi(x)$ means ' $x$ is an instance of (12)'. Each instance of (12) will follow. The strategy can be generalised just as before but using always Inf- $\mathrm{C}^{*}$ instead of Inf-C. 
It seems that, if no convincing reasons to admit introduction principles have been given yet, no convincing reasons to admit elimination principles have been given either. That is to say, both kinds of principles seem to have similar expressive power. ${ }^{13}$

\subsection{Reflection principles}

Picollo and Schindler address the following objection to their proposal.

A desirable feature of any theory of truth is that it admits the addition of its global reflection principle. Let $\Gamma$ be a recursively enumerable (r.e.) theory and $\operatorname{Prv}_{\Gamma}(x)$ a predicate that weakly represents provability in $\Gamma .{ }^{14}$ Then,

$$
\forall x\left(\operatorname{Prv}_{\Gamma}(x) \rightarrow T x\right)
$$

says that all theorems of $\Gamma$ are true. It allows us to express theoretical agreement or disagreement with theories that cannot be finitely axiomatised.

The problem is that, if $\Gamma$ is classical and supports an elimination principle, then its inner and outer logics disagree: ${ }^{15}$ given a liar sentence $\lambda,{ }^{16}$ we have both $\Gamma \vdash \lambda$ and $\Gamma \vdash \neg T\ulcorner\lambda\urcorner .{ }^{17}$ A consequence of this is that $\Gamma$ does not admit the addition of its global reflection principle. (For simplicity, the authors suppose that our syntax theory is Peano arithmetic (PA).)

Proposition 3 (Picollo and Schindler). Let $\Gamma \supseteq$ PA be a classical recursively enumerable (r.e.) theory. Let $\lambda$ be a liar sentence. If $\Gamma \vdash \lambda$, then $\Gamma+\mathrm{GRP}_{\Gamma}$ is inconsistent.

Nevertheless, the authors offer a solution. They argue that theoretical agreement can be expressed by the alternative sentence

$$
\forall x\left(\operatorname{Prv}_{\Gamma}(x) \rightarrow \neg T \neg x\right)
$$

which says that all theorems of $\Gamma$ are non-false. ${ }^{18}$ Then, they prove two results: that the-

\footnotetext{
${ }^{13}$ It is noteworthy that the counterarguments do not (and are not intended to) answer the objections A-D 'in their own spirit'. The counterarguments focus on the expressive functions of the truth predicate. They show that certain (sets of) sentences can be expressed by means of an elimination (introduction) principle alone. In contrast, the objections focus on the inferential roles of truth. They point to certain inferences that seem to be valid but, without suitable principles, do not hold. Since both Picollo and Schindler's paper and this essay focus strictly on the expressive functions of truth, this misappropriation of the objections does not constitute a problem.

${ }^{14} \mathrm{~A}$ formula $\phi(x)$ weakly represents a set $A$ in a theory $\Gamma$ just in case, for every object $a$ denoted by a term $\bar{a}$, we have $\Gamma \vdash \phi(\bar{a})$ if and only if $a \in A$.

${ }^{15}$ The outer logic of a theory $\Gamma \subseteq L_{T}$ is the set of its theorems. The inner logic is the set of sentences $\phi$ such that $\Gamma$ proves $T\ulcorner\phi\urcorner$.

${ }^{16}$ A liar is a sentence $\lambda$ s.t. our syntax theory proves $\lambda \leftrightarrow \neg T\ulcorner\lambda\urcorner$.

${ }^{17}$ Such disagreement could motivate some philosophical objections on its own (cf. Horsten 2011, p. 127; Leitgeb 2007, p. 282). Since those objections are not strictly concerned with the expressive functions of $T$, we do not address them here.

${ }^{18}$ If we regard "All theorems of $\Gamma$ are true" as somehow similar to "All theorems of $\Gamma$ are non-false", it is probably because of the intuitive validity of the rule that commutes truth and negation:

$$
\neg T\ulcorner\phi\urcorner \dashv \vdash T\ulcorner\neg \phi\urcorner
$$
}

Now, the left-to-right direction of this rule together with an elimination principle imply transparency; if the background logic is classical, this means triviality. Thus, the intuitive appeal of GRP* seems to rely on a rule that is not (fully) admissible in the framework. 
ory $\Gamma+\mathrm{GRP}_{\Gamma}^{*}$ is consistent, and that, under certain assumptions, $\Gamma+\mathrm{GRP}_{\Gamma}^{*}$ can prove the consistency of $\Gamma$.

Proposition 4 (Picollo and Schindler). If $\Gamma \supseteq$ PA contains T-Out and has a standard model (viz. an $\omega$-model) then $\Gamma+\mathrm{GRP}_{\Gamma}^{*}$ has a standard model too.

Proposition 5 (Picollo and Schindler). If $\Gamma \vdash T\ulcorner 0 \neq 1\urcorner$, then $\Gamma+\operatorname{GRP}_{\Gamma}^{*} \vdash \operatorname{Con}(\Gamma)$.

These results give rise to a question. Let $\Gamma$ be a classical theory of truth supporting at least an introduction principle. Does $\Gamma$ admit the addition of any variant of its global reflection principle?

Luckily, the introduction-based proposal lives up to our expectations. Indeed, it seems to slightly outperform its rival. The reason is that we can add the global reflection principle in its ordinary form. The inner and the outer logics of $\Gamma$ disagree: we have both $\Gamma \vdash \neg \lambda$ and $\Gamma \vdash T\ulcorner\lambda\urcorner$. In absence of elimination principles, however, this discrepancy does not lead to contradiction when $\mathrm{GRP}_{\Gamma}$ is added.

Observation 6. If $\Gamma \supseteq$ PA contains $T$-In and has a standard model, then $\Gamma+\operatorname{GRP}_{\Gamma}$ has a standard model too.

Proof. Assume the opposite. Then, there must be a standard model $\mathcal{M}$ such that $\mathcal{M} \vDash \Gamma$ and $\mathcal{M} \vDash \exists x\left(\operatorname{Prv}_{\Gamma}(x) \wedge \neg T x\right)$. Since $\mathcal{M}$ is standard, there must be a sentence $\psi$ such that $\mathcal{M} \vDash \operatorname{Prv}_{\Gamma}(\ulcorner\psi\urcorner) \wedge \neg T\ulcorner\psi\urcorner$. By the semantics of conjunction, $\mathcal{M} \vDash \operatorname{Prv}_{\Gamma}(\ulcorner\psi\urcorner)$ and $\mathcal{M} \vDash \neg T\ulcorner\psi\urcorner$. But since $\mathcal{M}$ is standard, $\mathcal{M} \vDash \operatorname{Prv}_{\Gamma}(\ulcorner\psi\urcorner)$ implies $\Gamma \vdash \psi$. By T-In we get $\Gamma \vdash T\ulcorner\psi\urcorner$ and therefore $\mathcal{M} \vDash T\ulcorner\psi\urcorner$. This contradicts the claim that $\mathcal{M} \vDash \neg T\ulcorner\psi\urcorner$.

Moreover, $\Gamma+\mathrm{GRP}_{\Gamma}$ can also prove, under certain assumptions, the consistency of $\Gamma$ :

Observation 7. If $\Gamma \vdash \neg T\ulcorner 0=1\urcorner$ then $\Gamma+\mathrm{GRP}_{\Gamma} \vdash \operatorname{Con}(\Gamma)$.

Proof. We apply the rule of Universal Instantiation to sentence $\mathrm{GRP}_{\Gamma}$ and obtain $\Gamma+\mathrm{GRP}_{\Gamma} \vdash$ $\operatorname{Prv}_{\Gamma}(\ulcorner 0=1\urcorner) \rightarrow T\ulcorner 0=1\urcorner$. By assumption, $\Gamma \vdash \neg T\ulcorner 0=1\urcorner$. Then $\Gamma+\operatorname{GRP}_{\Gamma} \vdash \neg T\ulcorner 0=1\urcorner$ and, by Modus Tollens, $\Gamma+\operatorname{GRP}_{\Gamma} \vdash \neg \operatorname{Prv}_{\Gamma}(\ulcorner 0=1\urcorner)$.

These results suggest that, as regards the reflection principles, the introduction-based proposal is not only as good as its rival but, maybe, even slightly more appealing.

To finish, I show a result analogous to Proposition 3: a classical theory of truth $\Gamma$ that validates $\mathrm{T}$-In or T-Intro does not admit the addition of the alternative reflection principle GRP $_{\Gamma}^{*}$. Let $\lambda^{*}$ be a sentence s.t. PA $\vdash \lambda^{*} \leftrightarrow \neg T \neg \neg\left\ulcorner\lambda^{*}\right\urcorner$. By classical logic and either T-In or T-Intro,

$$
\lambda^{*} \rightarrow T\left\ulcorner\neg \neg \lambda^{*}\right\urcorner
$$

But by definition of $\lambda^{*}$ and the identity $\neg \neg\left\ulcorner\lambda^{*}\right\urcorner=\left\ulcorner\neg \neg \lambda^{*}\right\urcorner$ provable in PA,

$$
\lambda^{*} \rightarrow \neg T\left\ulcorner\neg \neg \lambda^{*}\right\urcorner
$$

Hence, $\Gamma \vdash \neg \lambda^{*}$. Now,

Observation 8. Let $\Gamma \supseteq$ PA be a classical r.e. theory. Let $\lambda^{*}$ be a sentence s.t. PA $\vdash \lambda^{*} \leftrightarrow$ $\neg T \neg \neg\left\ulcorner\lambda^{*}\right\urcorner$. If $\Gamma \vdash \neg \lambda^{*}$, then $\Gamma+\mathrm{GRP}_{\Gamma}^{*}$ is inconsistent. 
Proof. Assume $\Gamma \vdash \neg \lambda^{*}$. By definition of $\lambda^{*}$ and the identity $\neg \neg\left\ulcorner\lambda^{*}\right\urcorner=\left\ulcorner\neg \neg \lambda^{*}\right\urcorner$ provable in PA, we get $\Gamma \vdash T\left\ulcorner\neg \neg \lambda^{*}\right\urcorner$. Since $\Gamma$ is an r.e. theory extending PA, $\Gamma \vdash \operatorname{Prv}_{\Gamma}\left(\left\ulcorner\neg \lambda^{*}\right\urcorner\right)$. By $\mathrm{GRP}_{\Gamma}^{*}$ and Modus Ponens, $\Gamma+\mathrm{GRP}_{\Gamma}^{*} \vdash \neg T \neg\left\ulcorner\neg \lambda^{*}\right\urcorner$. By identity $\neg\left\ulcorner\neg \lambda^{*}\right\urcorner=\left\ulcorner\neg \neg \lambda^{*}\right\urcorner$ provable in PA, $\Gamma+\mathrm{GRP}_{\Gamma}^{*} \vdash \neg T\left\ulcorner\neg \neg \lambda^{*}\right\urcorner$. But we obtained earlier that $\Gamma \vdash T\left\ulcorner\neg \neg \lambda^{*}\right\urcorner$. Hence, $\Gamma+\mathrm{GRP}_{\Gamma}^{*}$ is inconsistent.

\subsection{Conservativity ${ }^{19}$}

Let $\phi(x)$ be a predicate of $\mathcal{L}_{T}$-sentences and suppose there are infinitely many $\phi$ s. Let $\tau$ be a truth-theoretic sentence. An intuitive requirement for $\tau$ to express the conjunction of all the $\phi \mathrm{s}$ is that $\tau$ proves no more things than the set of conditionals $\phi(\ulcorner\psi\urcorner) \rightarrow \psi$. (We also expect $\tau$ to prove no less things, so it follows that $\tau$ and the conditionals should deliver the same consequences.) In this section I assess whether this requirement motivates a strict preference between introduction and elimination principles.

As Picollo and Schindler note, it is not reasonable to hold the mentioned requirement unrestricted. Any sentence entails itself. Due to compactness considerations, however, in a finitary first-order theory we cannot expect in general that a universal generalisation such as Inf-C or Inf-C* will be entailed by the relevant set of conditionals. Given the requirement, then, we could not expect in general that Inf-C or Inf- $\mathrm{C}^{*}$ expresses an infinite conjunction in any such theory (p. 905).

Halbach (1999) adopts a weakened version of the requirement. He focuses on infinite conjunctions of $T$-free sentences. Let $\phi(x)$ be a predicate of $\mathcal{L}$-sentences, and suppose there are infinitely many $\phi$ s. Recall $\Sigma$ is our base theory. The author's criterion can be stated thus: $\tau$ expresses the infinite conjunction of the $\phi \mathrm{s}$ in a theory of truth $\Gamma \supseteq \Sigma$ just in case theories $\Gamma+\tau$ and $\Sigma+\{\phi(\ulcorner\psi\urcorner) \rightarrow \psi: \psi \in \mathcal{L}\}$ have the same $T$-free consequences, or equivalently, $\Gamma+\tau$ is a conservative extension of $\Sigma+\{\phi(\ulcorner\psi\urcorner) \rightarrow \psi: \psi \in \mathcal{L}\}$.

Suppose that $\Sigma$ is classical. Halbach proves that Inf-C expresses the conjunction of the $\phi \mathbf{s}$ in the theory which extends $\Sigma$ with the T-Schema restricted to $\mathcal{L}$ :

Proposition 9 (Halbach). Let $\Gamma \subseteq \mathcal{L}_{T}$ extend $\Sigma$ with just the T-Schema restricted to $\mathcal{L}$. Let $\phi(x)$ be a predicate of $\mathcal{L}$-sentences. Then, theories $\Sigma+\{\phi(\ulcorner\psi\urcorner) \rightarrow \psi: \psi \in \mathcal{L}\}$ and $\Gamma+\forall x(\phi(x) \rightarrow T x)$ have the same $T$-free consequences in classical logic.

Given this Proposition and arguments from Sects. 2.1 and 3.1, it is easy to show two further results. First, Inf-C also expresses the conjunction of the $\phi \mathrm{s}$ in the theory which extends $\Sigma$ with T-Out restricted to $\mathcal{L}$ (see Picollo and Schindler, p. 908). Hence, Halbach's criterion does not justify the idea that the infinitary function requires a transparency principle. Second, Inf-C* expresses the conjunction of the $\phi \mathrm{s}$ in the theory which extends $\Sigma$ with T-In restricted to $\mathcal{L}$ (I leave the proof to the reader). Hence, Halbach's criterion also does not justify the idea that elimination principles play a privileged role in the infinitary function.

One of the putative advantages of abandoning transparency was that we could obtain a type-free theory of truth (see Sect. 1). Hence, the reader may rise a question here. Let $\Gamma$ extend $\Sigma$ with either an elimination or an introduction principle unrestricted. Can $\Gamma$ express the infinite conjunction of the $\phi$ s according to Halbach's criterion? I give a positive answer:

${ }^{19} \mathrm{I}$ thank an anonymous reviewer for encouraging me to address the subject of this section. 
Proposition 10. Let $\Gamma \subseteq \mathcal{L}_{T}$ extend $\Sigma$ with just T-Out unrestricted. Let $\phi(x)$ be as before. Then, theories $\Sigma+\{\phi(\ulcorner\psi\urcorner) \rightarrow \psi: \psi \in \mathcal{L}\}$ and $\Gamma+\forall x(\phi(x) \rightarrow T x)$ have the same $T$-free consequences in classical logic.

Proposition 11. Let $\Gamma \subseteq \mathcal{L}_{T}$ extend $\Sigma$ with just T-In unrestricted. Let $\phi(x)$ be as before. Then, theories $\Sigma+\{\phi(\ulcorner\psi\urcorner) \rightarrow \psi: \psi \in \mathcal{L}\}$ and $\Gamma+\forall x(\phi(x) \rightarrow \neg T \neg x)$ have the same $T$-free consequences in classical logic.

(The proofs can be found in the Appendix.) If $\Sigma$ is classical, Halbach's criterion is met by a theory of truth consisting in either half of the T-Schema, restricted or unrestricted.

Now, Heck (2004, p. 331) advances an objection against Halbach's account. They point out that the conservativity result in Proposition 9 does not extend to pairs of truth-theoretic sentences expressing infinite conjunctions. Consider the sentences:

$$
\begin{gathered}
\forall x\left[\exists n\left(x=\left\ulcorner\neg \operatorname{Prf}_{\mathrm{PA}}(\mathbf{n},\ulcorner 0=1\urcorner)\right\urcorner\right) \rightarrow T x\right] \\
\forall x\left[T x \rightarrow\left[\forall n\left(x=\left\ulcorner\neg \operatorname{Prf}_{\mathrm{PA}}(\mathbf{n},\ulcorner 0=1\urcorner)\right\urcorner \rightarrow \operatorname{Prf}_{\mathrm{PA}}(\mathbf{n},\ulcorner 0=1\urcorner)\right)\right]\right]
\end{gathered}
$$

where $\operatorname{Prf}_{\mathrm{PA}}(x, y)$ means that $x$ is (the Gödel number of) a PA-proof of the sentence (with Gödel number) $y$. Sentence (13) says that every sentence of the form ' $n$ is not a proof of ' $0=1$ ', is true. Sentence (14) says that, for every $n$, if the sentence which says ' $n$ is not a proof of ' $0=1$ ' ' is true, then $n$ is not a proof of ' $0=1$ '. Let $\mathrm{PA}^{\mathrm{T}} \mathrm{PA}^{\mathrm{T}}$ extend PA with the T-Schema restricted to $\mathcal{L}$. Theory $\mathrm{PA}^{\mathrm{T}}+(13)+(14)$ is a non-conservative extension of PA. However, PA plus the $T$-free instances of these sentences is just PA.

It is worth noting that Heck's objection also applies to the proposals defined in Sects. 2.1 and 3.1. To illustrate, let $\mathrm{PA}^{\mathrm{E}}$ be the result of extending PA with T-Out. Sentence (13) is an instance of Inf-C, so by the criterion and results in Sect. 2.1, it expresses an infinite conjunction in $\mathrm{PA}^{\mathrm{E}}$. Now, let us replace (14) by

$$
\forall x\left[\neg T \neg x \rightarrow\left[\forall n\left(x=\left\ulcorner\neg \operatorname{Prf}_{\mathrm{PA}}(\mathbf{n},\ulcorner 0=1\urcorner)\right\urcorner \rightarrow \operatorname{Prf}_{\mathrm{PA}}(\mathbf{n},\ulcorner 0=1\urcorner)\right)\right]\right]
$$

By analogy with the criterion in Sect. 2.1, we say that a sentence $\forall x(\neg T \neg x \rightarrow \phi(x))$ expresses an infinite conjunction $\bigwedge\left\{\psi \rightarrow \phi(\ulcorner\psi\urcorner): \psi \in \mathcal{L}_{T}\right\}$ in a theory of truth $\Gamma$ if and only if everything deducible from the set $\left\{\psi \rightarrow \phi(\ulcorner\psi\urcorner): \psi \in \mathcal{L}_{T}\right\}$ in $\Gamma$ is also deducible from $\forall x(\neg T \neg x \rightarrow \phi(x))$. Hence, it is easy to show that $\left(14^{*}\right)$ expresses an infinite conjunction in $\mathrm{PA}^{\mathrm{E}}$. Moreover, T-Out is enough to complete Heck's proof. $\mathrm{PA}^{\mathrm{E}}+(13)+\left(14^{*}\right)$ is a non-conservative extension of $\mathrm{PA}$, but PA plus the $T$-free instances of these sentences is just PA. The reader can do a similar reasoning to check that the proposal in Sect. 3.1 is also prone to Heck's objection. ${ }^{20}$

We considered an intuitive requirement according to which a sentence expressing the conjunction of the $\phi$ s should not prove more things than the set of $\phi$ s itself. Halbach's criterionwhich intends to capture a reasonable version of the requirement - is met by a classical theory

\footnotetext{
${ }^{20}$ Heck advances another objection against Halbach's account. The author argues that the truth predicate serves certain quasi-logical expressive functions which (i) are not plausibly described as that of expressing infinite conjunctions and disjunctions and (ii) require principles stronger than disquotation. This may be pressing for Halbach. However, I do not assume either that what I called the infinitary function exhausts the expressive utility of the truth predicate, or that a disquotation principle provides "a complete description of truth" (Halbach 1999, p. 1). Hence, I reckon that Heck's objection does not affect per se the semidisquotational proposals addressed. It would be coherent to say that, while an introduction or elimination principle is sufficient to express infinite conjunctions and disjunctions, a complete theory of truth must ultimately appeal to principles which go beyond disquotation.
} 
of truth consisting in either half of the T-Schema. Heck's objection - which exploits a reasonable extension of the requirement - affects both semidisquotational proposals considered in the essay. At any case, we found no motivation to have a strict preference between introduction and elimination principles.

\section{The outcome}

In this essay I answered the challenge posed by Picollo and Schindler. I argued that, under the author's assumptions, introduction principles are just as good as elimination principles to fulfil the infinitary function.

Picollo and Schindler point out that rejecting transparency could have certain advantages: we obtain a type-free, classical theory which seems to fulfil some of the main expressive functions of truth. The results of this essay can be read as a conditional statement: if we want to reject transparency, then, as regards the infinitary function, there seem to be no reasons of logical nature to choose between introduction and elimination principles. They achieve quite the same (meta)theoretical results. There may be other kinds of considerations (e.g. semantic, pragmatic) that tip the scales in favour of one or another. However, we will have to leave their assessment for another day.

Acknowledgements Earlier versions of this paper have been presented in 2018 at the Work in Progress Seminar of the Buenos Aires Logic Group (Buenos Aires, Argentina), also in 2018 at the National University of Rosario (Rosario, Argentina), and in 2019 at the Mathematical Philosophy Seminar of the Munich Center for Mathematical Philosophy (Munich, Germany); I would like to thank the attendees of these talks for their valuable feedback. I am grateful to Bruno Da Ré, Lavinia Picollo, Lucas Rosenblatt, Thomas Schindler and Diego Tajer for their help and guidance in the preparation of this paper. Finally, I would like to thank an anonymous reviewer of this journal, whose suggestions contributed to a significant improvement of the original draft.

\section{Appendix}

Recall that $\mathcal{L}$ contains a term $\ulcorner\sigma\urcorner$ denoting $\sigma$ (perhaps via some coding) for each expression $\sigma$ of $\mathcal{L}_{T}$. Suppose our base theory $\Sigma \subseteq \mathcal{L}$ is classical. Let $\phi(y)$ be a predicate of sentences of $\mathcal{L}$.

Proof of Proposition 10. Define $\Sigma^{\prime}$ as $\Sigma+\{\phi(\ulcorner\psi\urcorner) \rightarrow \psi: \psi \in \mathcal{L}\}$ and define $\Gamma^{\prime}$ as $\Gamma+\forall x(\phi(x) \rightarrow$ $T x)$. We show that $\Sigma^{\prime}$ and $\Gamma^{\prime}$ have the same $T$-free consequences.

Let $\chi \in \mathcal{L}$. Assume that $\Sigma^{\prime} \vdash \chi$. By the argument from Sect. 2.1 and the fact that $\Sigma$ is classical, $\Gamma^{\prime} \vdash \chi$. For the converse, we will use the following lemma:

Lemma 12. Any model $\mathcal{M}$ of $\Sigma^{\prime}$ can be expanded to a model $\mathcal{M}^{+}$of $\Gamma^{\prime}$.

Proof. If $\Sigma^{\prime}$ has no models, the Lemma holds trivially. So, let $\mathcal{M}=\langle D, I\rangle$ be a model where $D$ is the domain and $I$ the interpretation function, and suppose that $\mathcal{M} \models \Sigma^{\prime}$. Define $\mathcal{M}^{+}=\left\langle D, I^{+}\right\rangle$to be the expansion of $\mathcal{M}$ s.t. $I^{+}(T)=\{I(\ulcorner\psi\urcorner): \mathcal{M}=\phi(\ulcorner\psi\urcorner)\}$. We prove that $\mathcal{M}^{+} \vDash \Gamma^{\prime}$. 
(a) Let $\delta \in \mathcal{L}_{T}$. Assume $\mathcal{M}^{+} \models T\ulcorner\delta\urcorner$. Then, $I(\ulcorner\delta\urcorner) \in\{I(\ulcorner\psi\urcorner): \mathcal{M}=\phi(\ulcorner\psi\urcorner)\}$, and hence $\mathcal{M} \models \phi(\ulcorner\delta\urcorner)$. Besides, $\mathcal{M} \models \phi(\ulcorner\delta\urcorner) \rightarrow \delta$, so $\mathcal{M} \models \delta$. $\mathcal{M}^{+}$expands $\mathcal{M}$, so $\mathcal{M}^{+} \models \delta$. Therefore, $\mathcal{M}^{+} \models T\ulcorner\delta\urcorner \rightarrow \delta$.

(b) Let $s$ be any map from the variables in $\mathcal{L}_{T}$ to $D$. Let $s^{\prime}$ differ from $s$ at most at variable $x$. Assume $\mathcal{M}^{+}, s^{\prime} \models \phi(x) . \phi(y)$ is a predicate of sentences of $\mathcal{L}$ and $\mathcal{M}^{+}$ expands $\mathcal{M}$. Thus, $s^{\prime}(x) \in\{I(\ulcorner\psi\urcorner): \mathcal{M} \models \phi(\ulcorner\psi\urcorner)\}$. Hence, $\mathcal{M}^{+}, s^{\prime} \models T x$. Therefore, $\mathcal{M}^{+}, s^{\prime} \models \phi(x) \rightarrow T x$. Map $s^{\prime}$ was arbitrary. Thus, $\mathcal{M}^{+}, s \models \forall x(\phi(x) \rightarrow T x)$. Besides, $\forall x(\phi(x) \rightarrow T x)$ is a sentence, so $\mathcal{M}^{+} \models \forall x(\phi(x) \rightarrow T x)$.

Assume that $\Gamma^{\prime} \vdash \chi$. Suppose, for reductio, that $\Sigma^{\prime} \nvdash \chi$. Then, there is a model $\mathcal{M}$ of $\Sigma^{\prime}$ s.t. $\mathcal{M} \not \models \chi$. By our Lemma, there is an expansion $\mathcal{M}^{+}$s.t. $\mathcal{M}^{+} \models \Sigma^{\prime} \cup \Gamma^{\prime} . \mathcal{M}^{+} \not \chi \chi$, because $\mathcal{M}^{+}$expands $\mathcal{M}$ and $\chi \in \mathcal{L}$. But $\mathcal{M}^{+} \models \chi$, because $\mathcal{M}^{+} \models \Gamma^{\prime}$. A contradiction. Hence, $\Sigma^{\prime} \vdash \chi$.

Proof of Proposition 11. Define $\Sigma^{\prime}$ as $\Sigma+\{\phi(\ulcorner\psi\urcorner) \rightarrow \psi: \psi \in \mathcal{L}\}$ and define $\Gamma^{\prime}$ as $\Gamma+\forall x(\phi(x) \rightarrow$ $\neg T \neg x)$. We show that $\Sigma^{\prime}$ and $\Gamma^{\prime}$ have the same $T$-free consequences.

Let $\chi \in \mathcal{L}$. Assume that $\Sigma^{\prime} \vdash \chi$. By the argument from Sect. 3.1 and the fact that $\Sigma$ is classical, $\Gamma^{\prime} \vdash \chi$. For the converse, we will use the following lemma:

Lemma 13. Any model $\mathcal{M}$ of $\Sigma^{\prime}$ can be expanded to a model $\mathcal{M}^{+}$of $\Gamma^{\prime}$.

Proof. Again, if $\Sigma^{\prime}$ has no models, the Lemma holds trivially. So let $\mathcal{M}=\langle D, I\rangle$ be a model and suppose that $\mathcal{M} \models \Sigma^{\prime}$. Define $\mathcal{M}^{+}=\left\langle D, I^{+}\right\rangle$to be the expansion of $\mathcal{M}$ s.t. $I^{+}(T)=$ $\left\{I(\ulcorner\psi\urcorner): \psi \in \mathcal{L}_{T}\right\} \backslash\{I(\ulcorner\neg \psi\urcorner): \mathcal{M} \models \phi(\ulcorner\psi\urcorner)\}$. We prove that $\mathcal{M}^{+} \vDash \Gamma^{\prime}$.

(a) Let $\delta \in \mathcal{L}_{T}$. Assume $\mathcal{M}^{+}=\delta$. Then, $I(\ulcorner\delta\urcorner) \notin\{I(\ulcorner\neg \psi\urcorner): \mathcal{M} \models \phi(\ulcorner\psi\urcorner)\}$. (Assume the opposite. Then, there is a $\xi \in \mathcal{L}$ s.t. $\delta$ is identical to $\neg \xi$ and $\mathcal{M} \models \phi(\ulcorner\xi\urcorner) . \mathcal{M} \models$ $\phi(\ulcorner\xi\urcorner) \rightarrow \xi$, so $\mathcal{M} \models \xi$. $\mathcal{M}^{+}$expands $\mathcal{M}$, so $\mathcal{M}^{+} \models \xi$. Thus, $\mathcal{M}^{+} \not \models \delta$. A contradiction.) Hence, $\mathcal{M}^{+} \models T\ulcorner\delta\urcorner$. Therefore, $\mathcal{M}^{+} \models \delta \rightarrow T\ulcorner\delta\urcorner$.

(b) Let $s$ be any map from the variables in $\mathcal{L}_{T}$ to $D$. Let $s^{\prime}$ differ from $s$ at most at variable $x$. Assume $\mathcal{M}^{+}, s^{\prime} \models \phi(x) . \phi(y)$ is a predicate of sentences of $\mathcal{L}$ and $\mathcal{M}^{+}$ expands $\mathcal{M}$. Thus, $I(\neg)\left(s^{\prime}(x)\right) \in\{I(\ulcorner\neg \psi\urcorner): \mathcal{M} \models \phi(\ulcorner\psi\urcorner)\}$. Hence, $\mathcal{M}, s^{\prime} \mid \vDash T \neg x$. Therefore, $\mathcal{M}, s^{\prime} \models \neg T \neg x$ and $\mathcal{M}, s^{\prime} \models \phi(x) \rightarrow \neg T \neg x$. Map $s^{\prime}$ was arbitrary. Thus, $\mathcal{M}^{+}, s \models \forall x(\phi(x) \rightarrow \neg T \neg x)$. Besides, $\forall x(\phi(x) \rightarrow \neg T \neg x)$ is a sentence, so $\mathcal{M}^{+} \models$ $\forall x(\phi(x) \rightarrow \neg T \neg x)$

The proof continues as the analogous part of the proof of Proposition 10, but using Lemma 13 instead of Lemma 12. 


\section{References}

Beall, J. (2009). Spandrels of truth. Oxford: Oxford University Press.

Cobreros, P., Egré, P., Ripley, D., \& van Rooij, R. (2013). Reaching transparent truth. Mind, 122, 841-866.

Da Ré, B. (2020). Structural weakening and paradoxes. Manuscript.

Field, H. (2008). Saving truth from paradox. New York: Oxford University Press.

Gupta, A. (1993). A critique of deflationism. Philosophical Topics, 21, 57-81.

Halbach, V. (1999). Disquotationalism and infinite conjunctions. Mind, 108, 1-22.

Halbach, V. (2011). Axiomatic theories of truth. Cambridge University Press.

Heck, R. K. (2004). Truth and disquotation. Synthese, 142, 317-352. (Originally published under the name "Richard G. Heck, Jr".)

Horsten, L. (2011). The tarskian turn: Deflationism and axiomatic truth. Cambridge: MIT press.

Horwich, P. (1998). Truth (2nd edition). Oxford: Blackwell.

Kripke, S. (1975). Outline of a theory of truth. Journal of Philosphy, 72, 690-716.

Leeds, S. (1978). Theories of reference and truth. Erkenntnis, 13, 111-129.

Leitgeb, H. (2007). What theories of truth should be like (but cannot be). Philosophy Compass, 2, 276-290.

Picollo, L., \& Schindler, T. (2017). Disquotation and infinite conjunctions. Erkenntnis, 83, 899-928.

Picollo, L., \& Schindler, T. (2019). Deflationism and the function of truth. Philosophical Perspectives, 32, 326-351.

Priest, G. (2006). In contradiction. New York: Oxford University press.

Putnam, H. (1978). Meaning and the moral sciences. London: Routledge.

Quine, W. V. O. (1970). Philosophy of logic. Cambridge: Harvard University Press.

Rosenblatt, L. (2019). Noncontractive classical logic. Notre Dame Journal of Formal Logic, 60, $559-585$.

Tarski, A. (1935). The concept of truth in formalized languages. In J. Corcoran (Ed.), Logic, semantics, metamathematics (pp. 152-278). Oxford: Clarendon press.

Zardini, E. (2011). Truth without contra(di)ction. Review of symbolic logic, 4, 498-535. 